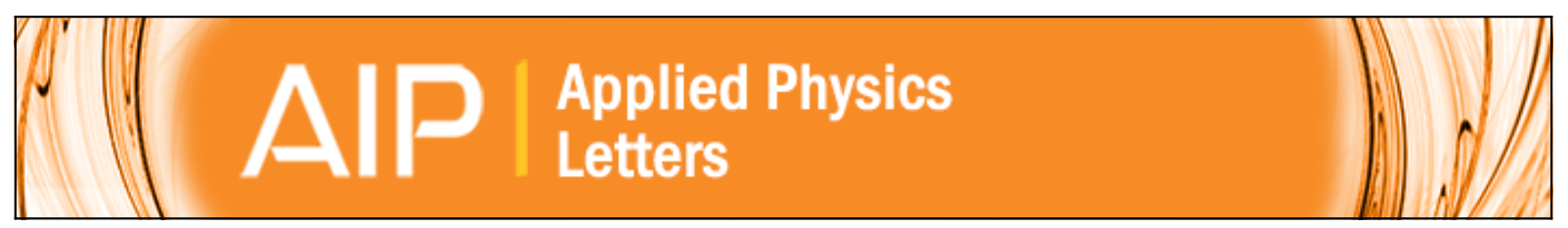

\title{
Electro optical tuning of Tamm-plasmon exciton-polaritons
}

J. Gessler, V. Baumann, M. Emmerling, M. Amthor, K. Winkler, S. Höfling, C. Schneider, and M. Kamp

Citation: Applied Physics Letters 105, 181107 (2014); doi: 10.1063/1.4901023

View online: http://dx.doi.org/10.1063/1.4901023

View Table of Contents: http://scitation.aip.org/content/aip/journal/apl/105/18?ver=pdfcov

Published by the AIP Publishing

\section{Articles you may be interested in}

Single-wavelength, all-optical switching based on exciton-polaritons

Appl. Phys. Lett. 101, 131104 (2012); 10.1063/1.4754575

Tuneable polaritonics at room temperature with strongly coupled Tamm plasmon polaritons in metal/air-gap microcavities

Appl. Phys. Lett. 98, 231105 (2011); 10.1063/1.3597304

Emission of Tamm plasmon/exciton polaritons

Appl. Phys. Lett. 95, 151114 (2009); 10.1063/1.3251073

Ultranarrow polaritons in a semiconductor microcavity

Appl. Phys. Lett. 76, 3262 (2000); 10.1063/1.126601

Collapse and revival of exciton-polariton oscillation in a semiconductor microcavity

Appl. Phys. Lett. 71, 1461 (1997); 10.1063/1.119936

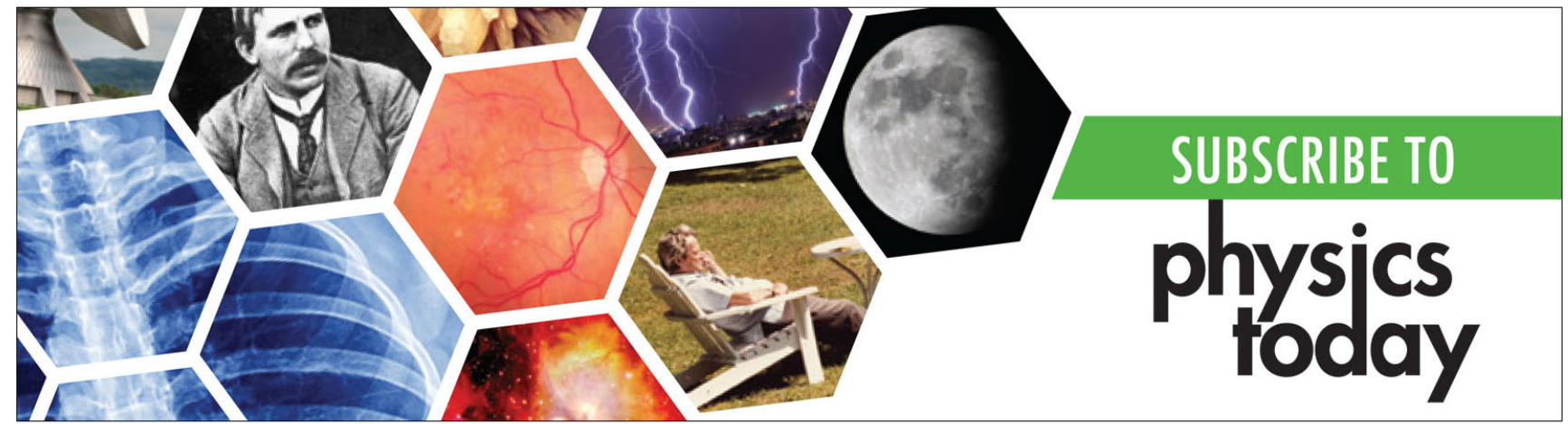




\title{
Electro optical tuning of Tamm-plasmon exciton-polaritons
}

\author{
J. Gessler, ${ }^{1}$ V. Baumann, ${ }^{1}$ M. Emmerling, ${ }^{1}$ M. Amthor,${ }^{1}$ K. Winkler, ${ }^{1}$ S. Höfling,${ }^{1,2}$ \\ C. Schneider, ${ }^{1}$ and M. Kamp ${ }^{1}$ \\ ${ }^{1}$ Technische Physik, Physikalisches Institut and Wilhelm Conrad Röntgen-Research Center for Complex \\ Material Systems, Universität Würzburg, Am Hubland, D-97074 Würzburg, Germany \\ ${ }^{2}$ SUPA, School of Physics and Astronomy, University of St. Andrews, St. Andrews, KY16 9SS, United Kingdom
}

(Received 5 September 2014; accepted 22 October 2014; published online 5 November 2014)

\begin{abstract}
We report on electro optical tuning of the emission from GaAs quantum wells resonantly coupled to a Tamm-plasmon mode in a hybrid metal/dielectric structure. The structures were studied via momentum resolved photoluminescence and photoreflectance spectroscopy, and the surface metal layer was used as a top gate, which allowed for a precise tuning of the quantum well emission via the quantum confined Stark effect. By tuning the resonance, we were able to observe the characteristic anticrossing behavior of a polaritonic emission in the strong light-matter coupling regime, yielding a Rabi splitting of $(9.2 \pm 0.2) \mathrm{meV}$. ( 2014 AIP Publishing LLC.

[http://dx.doi.org/10.1063/1.4901023]
\end{abstract}

Exciton-polaritons are bosonic quasiparticles composed of excitons and photons, which reveal rich and compelling physics suitable for studying collective phenomena of Bose gases in semiconductors. ${ }^{1,2}$ Their effective mass is about $10^{-5} m_{e}$ ( $m_{e}$ being the free electron mass), and their critical temperature for Bose-Einstein condensation (BEC) can therefore exceed $300 \mathrm{~K}$, which makes them very appealing to study bosonic condensation up to room temperature ${ }^{3-5}$ and allows observing fascinating related effects such as superfluidity $^{6}$ and the formation of vortices and solitons. ${ }^{7,8}$

A model-system to implement this vivid physics is given by quantum wells (QWs) integrated into a high-quality microcavity between two dielectric Bragg mirrors (DBRs), where the formation of exciton-polaritons can be established via the interaction of microcavity photons with QW-excitons in the strong coupling regime. In addition to several well-studied structures, strong light-matter interaction has also been reported for QW-excitons resonantly coupled to a Tammplasmon mode inside a hybrid metal/dielectric structure. ${ }^{9}$

Tamm-plasmon (TP) modes appear as localized electromagnetic modes at the boundary between a metal layer and a DBR analogous to purely electronic Tamm-states evolving at a crystal surface. ${ }^{10}$ Since the first experimental observation of TP-modes by Sasin et al., ${ }^{11}$ various structures based on this fascinating new type of excitation have been theoretically proposed, anticipating, e.g., resonant coupling of TPpolariton states to optical microcavity modes, ${ }^{12,13}$ nonlinear effects, such as optical bistability due to field enhancement in hybrid metal/dielectric structures, ${ }^{14}$ or integrated circuits based on plasmon-exciton-polaritons. ${ }^{15}$ Experimentally, laser oscillation from hybrid metal/semiconductor structures has been observed. ${ }^{16}$

Similar to Fabry-Perot cavity resonances, TP-modes appear as sharp dips in the photoreflectance spectra and show a parabolic in-plane dispersion of the photons. Additionally, the eigenenergies of the resonant photons depend both on the thickness of the metal film and the thickness of the adjacent dielectric layer, which can be easily designed to match a resonance across almost the entire photonic band gap of the DBR. ${ }^{10}$ The tight localization of the electromagnetic field, which is essential for the observation of light-mater interaction in the strong-coupling regime, is achieved due to the negative refractive index of the metal on the one side and due to the photonic band gap of the DBR on the other side of the interface. In addition, the fabrication of these structures is relatively simple.

A further advantage of such hybrid metal/dielectric structures is their tunability, which arises from the close proximity of the active layer and the metal film. The latter can be easily utilized as an electric contact with high optical transmission, as the TP-mode provides a pathway for resonant photon tunneling. ${ }^{17}$ In this work, we take full advantage of this approach and present an electrostatic tunable device based on a hybrid metal/DBR structure, embedding QWs coupled to a TP-mode for operation in the strong coupling regime.

The investigated samples were fabricated on an n-doped GaAs (001) substrate with a dopant concentration of $5 \times 10^{18} \mathrm{~cm}^{-3}$ by solid-source molecular beam epitaxy. The epitaxial structure is made up of a bottom DBR and a top layer embedding 3 multiple QW stacks. The DBR consists of $20.5 \mathrm{Al}_{0.2} \mathrm{Ga}_{0.8} \mathrm{As} / \mathrm{AlAs}$ mirror pairs, where the first $18.5 \mathrm{mir}-$ ror pairs were $\mathrm{n}$-doped with a dopant concentration of $1 \times 10^{18} \mathrm{~cm}^{-3}$, dropping exponentially to $5 \times 10^{17} \mathrm{~cm}^{-3}$ over the last two mirror pairs. The thicknesses of the DBR-layers were designed to match the photonic band gap and the QWemission at the center of the wafer. The top layer consists of $\mathrm{Al}_{0.2} \mathrm{Ga}_{0.8} \mathrm{As}$ and contains a total of eight GaAs QWs in a 2/4/ 2 configuration emitting at $1.598 \mathrm{eV}$ at $10 \mathrm{~K}$. The thickness of this layer was designed to match the eigenenergy of the TPmode and the QW-emission. The QWs were placed at the antinodes of the electromagnetic field to enhance the lightmatter interaction. In order to improve the carrier confinement and to suppress undesired tunnel losses in the presence of an electric field, the QWs are separated by $4 \mathrm{~nm}$ thick AlAs barriers. The structure was capped by $10 \mathrm{~nm}$ GaAs to avoid oxidation. In Fig. 1(a), a cross-section scanning electron microscope image of the structure is depicted. After growth, a $40 \mathrm{~nm}$ thick $\mathrm{SiO}_{2}$ isolation-layer was deposited on top of the structure to suppress current leakage when a voltage is applied 


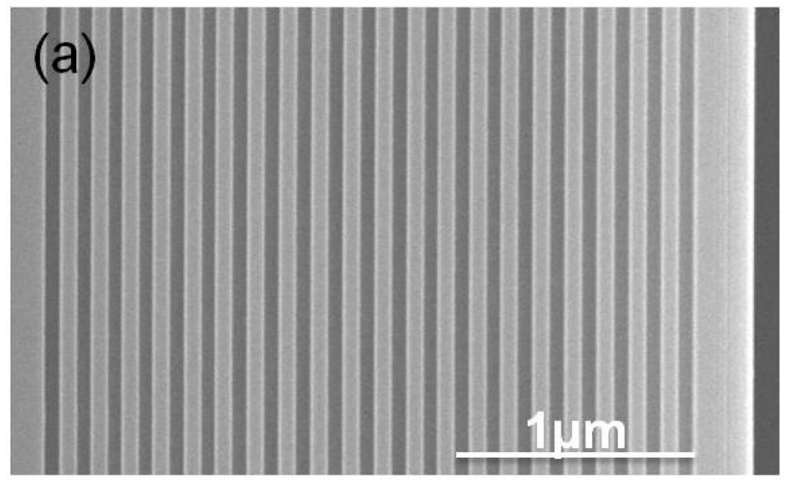

(c)

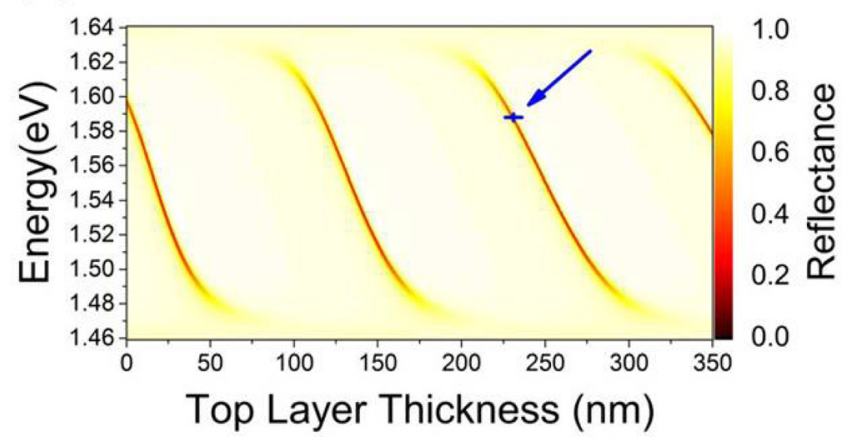

(b)
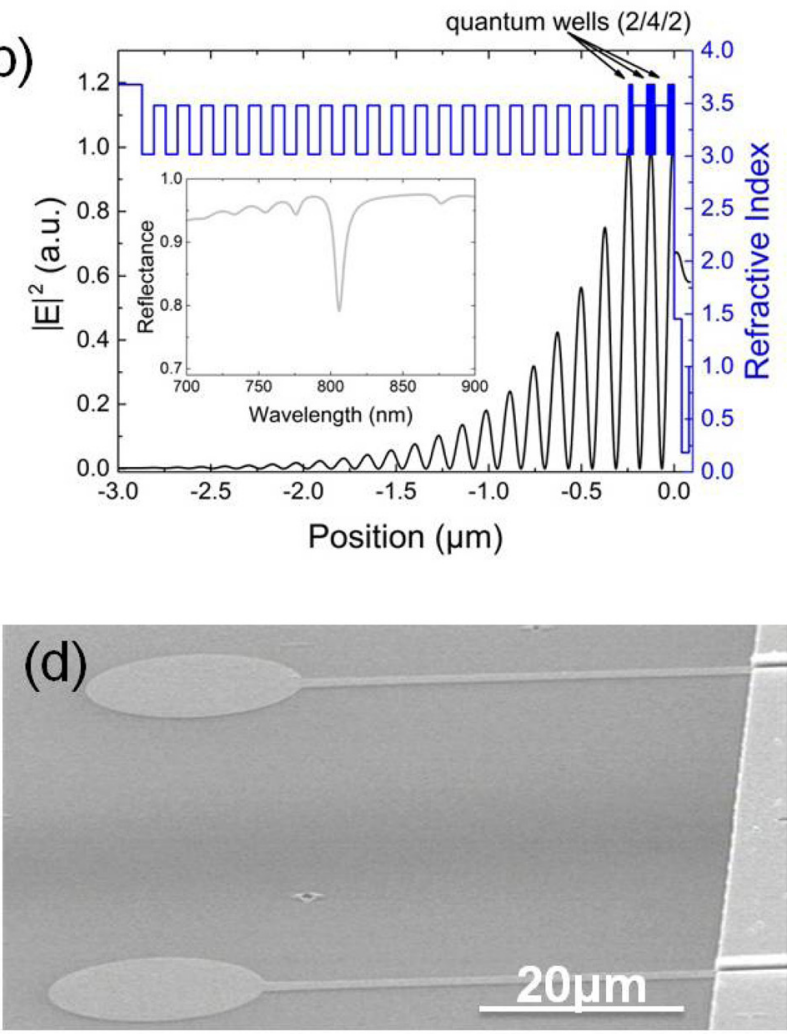

FIG. 1. (a) Scanning electron microscopy (SEM) image of grown structure, consisting of a bottom DBR with $20.5 \mathrm{Al}_{0.2} \mathrm{Ga}_{0.8} \mathrm{As} / \mathrm{AlAs}$ mirror pairs and an $\mathrm{Al}_{0.2} \mathrm{Ga}_{0.8} \mathrm{As}$ top layer with eight embedded GaAs QWs. (b) Calculated intensity profile of the electric field inside the structure. On the right scale (blue), the refractive index profile of the correspondent layers is displayed. The inset displays the calculated reflectivity spectrum. (c) Calculated reflectivity spectra as a function of the top layer thickness, showing the TP-mode, which shifts to lower energies with increasing thickness and reappears periodically, when the thickness is further increased. The blue marking and arrow indicate the spectral position of the TP-mode derived from experimental data for the given thickness of the top layer in the actual structure. (d) Surface SEM image of the structured sample, showing electrically contactable $20 \mu \mathrm{m}$ gold discs for Stark tuning experiments.

across the sample. Finally, a $40 \mathrm{~nm}$ thick gold (Au) film was deposited on the surface of the structure by thermal evaporation. In Fig. 1(b), the refractive index profile of the structure is shown together with the expected intensity of the electric field, which was calculated by eigenmode expansion using the software CAMFR. ${ }^{18}$ The inset shows the calculated reflectivity spectrum of the structure, which reveals the characteristic optical resonance corresponding to the TP-mode. In Fig. 1(c), the expected evolution of the TP-mode is shown as a function of the thickness of the topmost dielectric layer, which was calculated using the transfer matrix method. It can be clearly seen that the TP-mode shifts across the photonic band gap of the DBR with the increase in thickness of the top layer, and modes reappear periodically when the thickness is further increased, which agrees with previous reports. ${ }^{19}$ The periodic appearance of the TP-mode is very useful, as it allows to design the top layer with a sufficient thickness to embed several active layers at the respective maxima of the field intensity. Further, the decrease of the layer thickness in the outward radial direction of the wafer can be exploited to spectrally tune the TP-resonance. An unstructured sample with a planar Au-film on top of the $\mathrm{SiO}_{2}$ layer was used for basic characterization. For the Stark tuning experiments, metal discs with a diameter of $20 \mu \mathrm{m}$ were defined on the $\mathrm{SiO}_{2}$ layer by electron beam lithography, evaporation of a $40 \mathrm{~nm}$ gold layer and lift off. The discs are connected to a bond pad by thin Au-stripes as shown in Fig. 1(d).
First, we have investigated the unstructured sample, covered by a continuous gold layer. We measured the photoluminescence (PL) in a Fourier space PL setup with micrometer spatial resolution. This setup is composed of a standard micro-PL configuration with an additional far field lens to convert the real space image into the momentum-space (k-space) image. ${ }^{20}$ It allows a direct acquisition of the momentum-energy dispersion by imaging the Fourier plane on a two-dimensional CCD sensor. We recorded the PL of the sample, carried out under non-resonant excitation $(920 \mu \mathrm{W})$ at a sample temperature of $6 \mathrm{~K}$, as a function of the wafer position in $0.5 \mathrm{~mm}$ steps and extracted line spectra from the data at $\mathrm{k}=(0.0 \pm 0.1) \mu \mathrm{m}^{-1}$. In Figs. 2(a) and 2(b), one can see the PL-spectra, taken at different positions of the wafer and the analysis of the emission energy and linewidth (inset) of the corresponding peaks. A clear evidence of the characteristic anticrossing is observable with a Rabi splitting $E_{R S}=(8.0 \pm 0.3) \mathrm{meV}$ at $6.0 \mathrm{~mm}$ distance from the center of the wafer (Fig. 2(b)). We also observe an exchange of the linewidth of the two polariton peaks (inset Fig. 2(b)) which supports our interpretation of the experiment as operation in the strong coupling regime. We further note the presence of the unbound exciton which remains unaffected in energy and linewidth throughout this resonance tuning experiment, which is in agreement with previous reports. ${ }^{21}$

In order to investigate the electro-optical tuning characteristics of our structure, we studied the sample with the electrically 
(a)

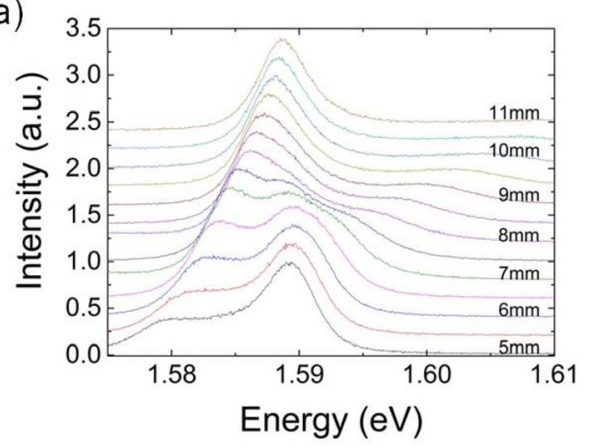

(b)

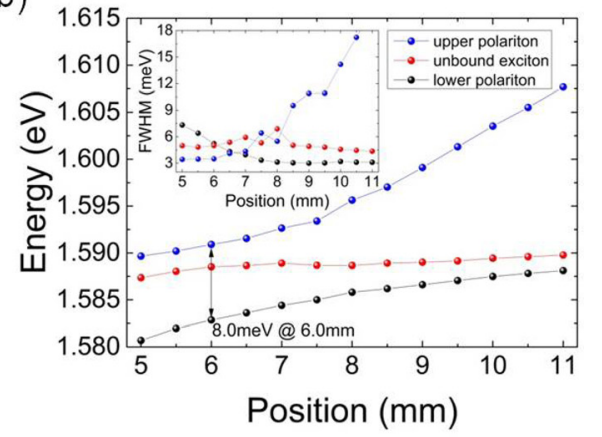

FIG. 2. (a) Photoluminescence spectra taken at different positions of the unstructured sample. (b) Analysis of the spectra taken in Fig. 2(a) after fitting with three Lorentzian peaks. The black and blue data points correspond to the lower and upper polariton branch. The red data points are attributed to the uncoupled exciton. contacted gold discs in greater detail. We first discuss the voltage/current characteristic of the device. The current through the sample was determined from the voltage drop across a $1 \mathrm{k} \Omega$ series resistance which was inserted between the voltage source and the sample. Negligible leakage current was observed for voltages in the range from $-5 \mathrm{~V}$ to $+7 \mathrm{~V}$.

For Stark tuning measurements, the sample was held at $6 \mathrm{~K}$ and investigated via white light reflection. A pinhole in the focal plane of the far field lens was introduced for spatial selection of the signal stemming from one $20 \mu \mathrm{m}$ disc. Fig. 3(a) displays the momentum resolved spectrum at different applied voltages. The exciton-photon detuning for this particular disc was determined to be $-9.5 \mathrm{meV}$ at zero applied voltage. From the linewidth of the polariton resonances, we can extract a quality factor of 350 . With increasing voltage in reverse direction, the exciton is subject to a spectral redshift, as one can extract from the measurement carried out at $+3.0 \mathrm{~V}$ and $+5.8 \mathrm{~V}$ (indicated by the white arrows). Fig. 3(b) shows the analysis of the complete electrical tuning experiment. The upper and lower polariton branch are subject to a distinct redshift and exhibit a Rabi splitting of
$\mathrm{E}_{\mathrm{RS}}(9.2 \pm 0.2) \mathrm{meV}$, which slightly exceeds the value determined by the spatial variation of the detuning as a consequence of modest carrier screening effects. ${ }^{22}$ From the extracted Rabi splitting, we can assess the oscillator strength per unit area of our QWs, which is in good agreement with previous reports $^{23}$

$$
f_{e x}=\frac{L_{e f f} m_{e} n_{c a v}^{2} \varepsilon_{0} E_{R S}^{2}}{2 \hbar^{2} e_{0}^{2} N_{Q W}}=(6.45 \pm 0.14) \times 10^{12} \mathrm{~cm}^{-2} .
$$

Here, $L_{\text {eff }}(1.41 \mu \mathrm{m})$ denotes the effective cavity length and $\mathrm{E}_{\mathrm{RS}}$ the Rabi-splitting.

We can calculate the bare TP-mode $\left(\mathrm{E}_{\mathrm{TP}}\right)$ and exciton mode $\left(\mathrm{E}_{\text {exc }}\right)$ from the measured data using the extracted Rabi splitting $E_{R S}$ with $E_{L P}$ and $E_{U P}$ being the energy of the lower and upper polariton branch, respectively ${ }^{24}$

$$
E_{T P / e x c}=\frac{1}{2}\left(E_{L P}+E_{U P} \pm \sqrt{E_{L P}^{2}-2 E_{L P} E_{U P}+E_{U P}^{2}-E_{R S}^{2}}\right) .
$$

The resulting spectral position of the bare TP-mode for the given thickness of the top layer is in excellent agreement
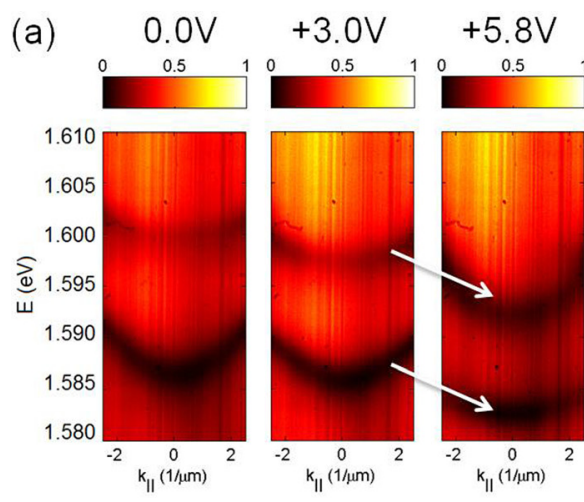

(b)

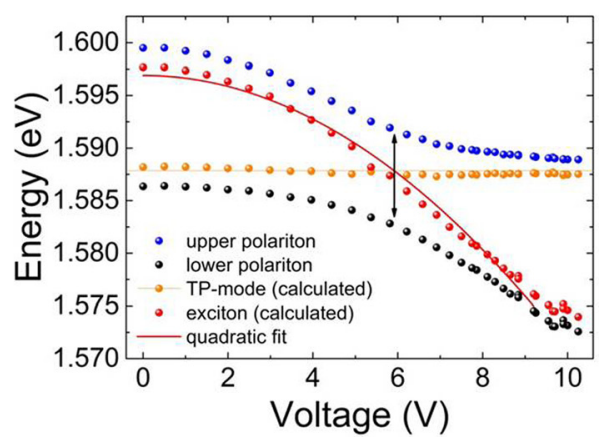

(c)

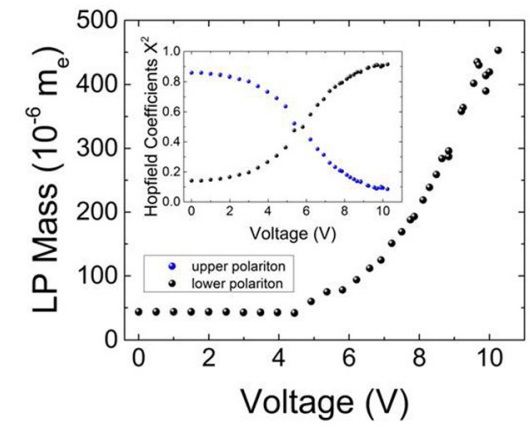

FIG. 3. (a) Momentum resolved photoreflectance spectra from a $20 \mu \mathrm{m}$ gold disc. The images show the dispersion recorded at the indicated voltages. With the increase in voltage, the emission shifts red while the dispersion of the lower branch flattens. Correspondingly, the curvature of the dispersion of the upper branch increases. (b) Analysis of the measurements taken in Fig. 3(a). For positive voltages, the Stark tuning reveals the anticrossing with a Rabi splitting of $(9.2 \pm 0.2) \mathrm{meV}$. The bare TP-mode and exciton are calculated out of the measured data. The quadratic dependency of the exciton energy to the external voltage is fitted by the continuous red line. (c) Polariton effective masses and the Hopfield coefficients (inset) as a function of the applied gate voltage. 
with the simulation in Fig. 1(c). From the trace of the uncoupled exciton energy (depicted in Fig. 3(b)), we can derive a spectral exciton shift of $\Delta \mathrm{E}=(-0.259 \pm 0.005) \frac{\mathrm{meV}}{\mathrm{V}^{2}} \times U^{2}$ (where $U$ is the voltage applied to the sample), which is in good agreement with the directly calculated value $^{25}$ of $(-0.258 \pm 0.010) \frac{\mathrm{meV}}{\mathrm{V}^{2}}$ for the polarizability of the $\mathrm{QW}$, and excludes thermal emission shifts via current heating as the source of the tuning behavior.

As a consequence of the distinct change of the detuning, we observe a strong modification of the curvature of the polariton branches (Fig. 3(a)). The extracted effective masses of the lower polaritons (derived by approximating the dispersion with a parabola in the range from $\mathrm{k}=-0.8 \mu \mathrm{m}^{-1}$ to $\mathrm{k}=0.8 \mu \mathrm{m}^{-1}$ ) are shown in Fig. 3(c)). In fact, we can modify the effective mass of our polaritons via Stark tuning by more than one order of magnitude. The according Hopfield coefficients $^{26} \mathrm{X}^{2}$ and $\mathrm{C}^{2}$, derived from the measured photon and exciton energies and the given Rabi splitting, are plotted in the inset of Fig. 3(c). This furthermore demonstrates the capability to tune our system from highly excitonic to highly photonic character via the quantum confined Stark effect.

In conclusion, we present complete electro-optical resonance tuning of QWs coupled to a TP-mode in a hybrid metal/dielectric structure, operated in the strong coupling regime. The resonance tuning via the quantum confined Stark effect was studied in detail, revealing a Rabi-splitting of $(9.2 \pm 0.2) \mathrm{meV}$, a spectral shift of the exciton caused by the external field of $\Delta \mathrm{E}=-0.259 \frac{\mathrm{meV}}{\mathrm{V}^{2}} \times U^{2}$ and a oscillator strength of $(6.45 \pm 0.14) \times 10^{12} \mathrm{~cm}^{-2}$ for the GaAs QWs embedded in the $\mathrm{Al}_{0.2} \mathrm{Ga}_{0.8} \mathrm{As}$ layer. We anticipate that such electrically tunable hybrid structures can play a crucial role towards the implementation in polaritonic circuits, switches, and ultrafast solitonic devices. We furthermore highlight the possibility to define electro-optical traps and potentials via the discussed technique, which allows to design tunable potential landscapes for polaritons and their condensates in future experiments.

This work was financially supported by the state of Bavaria, the bilateral project of the Deutsche Forschungsgemeinschaft (within the project LIEPOLATE) and the Polish Ministry of Science and higher education (Project No. DPN/N99/DFG/2010) and the EU within the project SPANGL4Q.
${ }^{1}$ I. Carusotto and C. Ciuti, Rev. Mod. Phys. 85, 299-366 (2013).

${ }^{2}$ A. Kavokin and G. Malpuech, Cavity Polaritons (Elsevier Academic Press, 2003).

${ }^{3}$ S. Christopoulos, G. B. H. von Högersthal, A. J. D. Grundy, P. G. Lagoudakis, A. V. Kavokin, J. J. Baumberg, G. Christmann, R. Butté, E. Feltin, J.-F. Carlin, and N. Grandjean, Phys. Rev. Lett. 98, 126405 (2007). ${ }^{4}$ T.-C. Lu, Y.-Y. Lai, Y.-P. Lan, S.-W. Huang, J.-R. Chen, Y.-C. Wu, W.-F. Hsieh, and H. Deng, Opt. Express 20, 5530-5537 (2012).

5 J. D. Plumhof, T. Stöferle, L. Mai, U. Scherf, and R. F. Mahrt, Nat. Mater. 13, 247-252 (2014).

${ }^{6}$ K. S. Daskalakis, S. A. Maier, R. Murray, and S. Kéna-Cohen, Nat. Mater. 13, 271-278 (2014).

${ }^{7}$ K. Lagoudakis, M. Wouters, M. Richard, A. Baas, I. Carusotto, R. André, L. S. Dang, and B. Deveaud-Plédran, Nat. Phys. 4, 706-710 (2008).

${ }^{8}$ A. Amo, D. Sanvitto, F. P. Laussy, D. Ballarini, E. del Valle, M. D. Martin, A. Lemaître, J. Bloch, D. N. Krizhanovskii, M. S. Skolnick, C. Tejedor, and L. Viña, Nature 457, 291-295 (2009).

${ }^{9}$ C. Symonds, A. Lemaître, E. Homeyer, J. C. Plenet, and J. Bellessa, Appl. Phys. Lett. 95(15), 151114 (2009).

${ }^{10}$ M. Kaliteevski, I. Iorsh, S. Brand, R. A. Abram, J. M. Chamberlain, A. V. Kavokin, and I. A. Shelykh, Phys. Rev. B 76, 165415 (2007).

${ }^{11}$ M. E. Sasin, R. P. Seisyan, M. A. Kaliteevski, S. Brand, R. A. Abram, J. M. Chamberlain, A. Yu. Egorov, A. P. Vasil'ev, V. S. Mikhrin, and A. V. Kavokin, Appl. Phys. Lett. 92, 251112 (2008).

${ }^{12}$ Y. Fang, L. Yang, W. Kong, and N. Zhu, J. Opt. 15, 125703 (2013).

${ }^{13}$ M. Kaliteevski, S. Brand, R. A. Abram, I. Iorsh, A. V. Kavokin, and I. A. Shelykh, Appl. Phys. Lett. 95, 251108 (2009).

${ }^{14}$ K. J. Lee, J. W. Wu, and K. Kim, Opt. Express 21(23), 28817 (2013).

${ }^{15}$ T. C. H. Liew, A. V. Kavokin, T. Ostatnický, M. Kaliteevski, I. A. Shelykh, and R. A. Abram, Phys. Rev. B 82, 033302 (2010).

${ }^{16}$ C. Symonds, G. Lheureux, J. P. Hugonin, J. J. Greffet, J. Laverdant, G. Brucoli, A. Lemaitre, P. Snellart, and J. Bellessa, Nano Lett. 13, 3179 (2013).

${ }^{17}$ K. Leosson, S. Shayestehaminzadeh, T. K. Tryggvason, A. Kossoy, B. Agnarsson, F. Magnus, S. Olafsson, J. T. Gudmundsson, E. B. Magnusson, and I. A. Shelykh, Optics Lett. 37, 4026 (2012).

${ }^{18} \mathrm{P}$. Bienstman and R. Baets, Opt. Quantum Electron. 33, 327-341 (2001).

${ }^{19}$ H. Zhou, G. Yang, K. Wang, H. Long, and P. Lu, Opt. Lett. 35, 4112 (2010).

${ }^{20}$ C. W. Lai, N. Y. Kim, S. Utsunomiya, G. Roumpos, H. Deng, M. D. Fraser, T. Byrnes, P. Recher, N. Kumuda, T. Fujisawa, and Y. Yamamoto, Nature 450, 529-532 (2007).

${ }^{21}$ M. C. Huang, Y. Zhou, and C. J. Chang-Hasnain, Nat. Photon. 1(2), 119-122 (2007).

${ }^{22}$ R. Houdré, J. L. Gibernon, P. Pellandini, R. P. Stanley, U. Oesterle, C. Weisbuch, J. O'Gorman, B. Roycroft, and M. Ilegems, Phys. Rev. B 52(11), 7810 (1995).

${ }^{23}$ L. C. Andreani and A. Pasquarello, Phys. Rev. B 42(14), 8928-8937 (1990).

${ }^{24}$ H. Deng, H. Haug, and Y. Yamamoto, Rev. Mod. Phys. 82(2), 1489 (2010).

${ }^{25}$ D. A. B. Miller, D. S. Chemla, T. C. Damen, A. C. Gossard, W. Wiegmann, T. H. Wood, and C. A. Burrus, Phys. Rev. Lett. 53(22), 2173-2176 (1984).

${ }^{26}$ J. J. Hopfield, Phys. Rev. 112, 1555 (1958). 